\title{
Hormona de crecimiento en sujetos varones de diferentes altitudes
}

\author{
Growth hormone in males at different altitude
}

\author{
Elydia Mujica1a ${ }^{1 a}$ Haydée Zúñiga ${ }^{3 a}$, Hugo Cebreros $^{1 a}$, José Aliaga ${ }^{4 a}$, Gerardo Ronceros ${ }^{2 a}$, \\ José Ramos-Castillo ${ }^{2 b, 5 c}$, Javier Torres ${ }^{1 a}$, Walter Ponciano ${ }^{1 a}$, Nicolás López ${ }^{2 d}$, José Ortiz ${ }^{1 a}$, Yeliz Bravo ${ }^{1 a}$ \\ ${ }^{1}$ Instituto Nacional de Biología Andina, Facultad de Medicina, UNMSM. Lima, Perú. \\ ${ }^{2}$ Facultad de Medicina, UNMSM. Lima, Perú. \\ ${ }^{3}$ Facultad de Farmacia y Bioquímica, UNMSM. Lima, Perú. \\ ${ }^{4}$ Escuela de Farmacia y Bioquímica, UPCH. Lima, Perú. \\ ${ }^{5}$ Policlínico Fiori, Red Asistencial Sabogal, EsSalud. Lima, Perú. \\ a Docente, ${ }^{b}$ Egresado ${ }^{c}$ Médico-Cirujano, ${ }^{d}$ Egresado de la Maestría en Fisiología
}

\begin{abstract}
Resumen
Objetivos: Determinar los niveles de hormona de crecimiento (GH) en sujetos varones adultos, a nivel del mar (Lima, $150 \mathrm{msnm}$ ) y en la altura (Cajamarca: 2 750 msnm; Huancayo: 3280 msnm; Morococha: 4540 msnm). Diseño: Estudio descriptivo, observacional, transversal y prospectivo. Lugar: Ciudades de Lima, Cajamarca, Huancayo y Morococha, Perú. Participantes: Adultos varones, nativos de localidades de diferentes altitudes. Intervenciones: Previo consentimiento informado, a 41 adultos varones nativos de su localidad de origen [12 sujetos al nivel del mar (Lima $150 \mathrm{~m}$ ), 10 sujetos en Cajamarca (2 $750 \mathrm{~m}$ ), 12 sujetos en Huancayo (3 $280 \mathrm{~m}$ ) y 7 sujetos en Morococha (4 $540 \mathrm{~m}$ )] se tomó muestras de sangre en ayunas y en condiciones de reposo. Principales medidas de resultados: Niveles sanguíneos de hormona de crecimiento $(\mathrm{GH})$ en sangre. Resultados: La edad promedio de los participantes fue 28 años (rango de 20 a 35 años). Los valores promedios de GH encontrados fueron: a $4540 \mathrm{~m}, 3,87 \pm 0,16 \mathrm{ng} / \mathrm{mL}$; a $3280 \mathrm{~m}, 1,31 \pm 0,09 \mathrm{ng} / \mathrm{mL}$; a $2750 \mathrm{~m}, 0,63 \pm 0,08 \mathrm{ng} / \mathrm{mL}$; y a $150 \mathrm{~m}, 0,54$ $\pm 0,04 \mathrm{ng} / \mathrm{mL}$. Hubo diferencia significativa $(p<0,05)$ entre los valores de $\mathrm{GH}$ encontrados en la altura a $4540 \mathrm{~m}$ y $3280 \mathrm{~m}$ con respecto al nivel del mar 150 $\mathrm{m}$. No se encontró diferencia significativa entre los valores encontrados a $2750 \mathrm{~m}$ con respecto a $150 \mathrm{~m}$. Conclusiones: Los niveles de GH son mayores en los sujetos nativos a $3280 \mathrm{~m}$ (Huancayo, $\mathrm{n}=12$ ) y $4540 \mathrm{~m}$ (Morococha, $\mathrm{n}=7$ ) respecto a los sujetos que viven a $150 \mathrm{~m}$ (Lima, $\mathrm{n}=12$ ).

Palabras clave: Hormona de crecimiento humana; altitud; zonas geográficas.
\end{abstract}

\begin{abstract}
Objectives: To determine growth hormone (GH) serum levels in adult males at sea level (Lima, $150 \mathrm{msnm}$ ) and high altitude (Cajamarca: $2750 \mathrm{msnm}$, Huancayo: 3280 msnm, Morococha: 4540 msnm). Design: Descriptive, observational, transversal and prospective study. Location: Lima, Cajamarca, Huancayo y Morococha, Peru. Participants: Male adult natives of different altitude settings. Interventions: With informed consent blood samples were obtained from the forearm in fasting and resting conditions from 41 young adult male subjects 20 to 35 years old [ 12 subjects at sea level (Lima $150 \mathrm{~m}$ ), 10 subjects in Cajamarca (2 $750 \mathrm{~m}$ ), 12 subjects in Huancayo (3 $280 \mathrm{~m}$ ), and 7 subjects in Morococha (4 $540 \mathrm{~m}$ )]. Main outcome measures: Serum growth hormone (GH) levels. Results: GH mean values found were at $4540 \mathrm{~m}: 3,87 \pm 0,16 \mathrm{ng} / \mathrm{mL}$; at $3280 \mathrm{~m}$ : $1,31 \pm 0,09 \mathrm{ng} / \mathrm{mL}$; at $2750 \mathrm{m:} 0,63 \pm 0,08 \mathrm{ng} / \mathrm{mL}$; and at $150 \mathrm{m:} 0,54 \pm$ $0,04 \mathrm{ng} / \mathrm{mL}$. There was significant difference $(p<0,05)$ between values found at altitudes $4540 \mathrm{~m}$ and $3280 \mathrm{~m}$ above sea level $(150 \mathrm{~m})$. No significant difference was found between values found at $2750 \mathrm{~m}$ and $150 \mathrm{~m}$. Conclusions: Adult male growth hormone serum levels were higher at Huancayo ( $3280 \mathrm{msnm}, \mathrm{n}=12$ ) and Morococha (4 $540 \mathrm{msnm}, \mathrm{n=7}$ ) than at sea level (Lima $150 \mathrm{msnm}, \mathrm{n}=12$ ). Key words: Human growth hormone; altitude; geographic locations.
\end{abstract}

An Fac med. 2010;71(3):167-70

\section{INTRODUCCIÓN}

La hormona de crecimiento $(\mathrm{GH})$ es sintetizada y liberada de la hipófisis, bajo control hipotalámico de estimulación por la hormona liberadora de $\mathrm{GH}$ y de inhibición por la somatostatina. Muchos otros factores (hormonales y no hormonales) también modulan la síntesis, liberación y respuesta celular de la GH. Así, por ejemplo, los estrógenos, el glucagón, la hormona antidiurética, la grelina, la hipoglicemia, el ejercicio y el sueño incrementan los niveles de $\mathrm{GH}$. Por el contrario, los glucocorticoides, la $\mathrm{GH}$ exógena y los ácidos grasos libres inhiben la liberación de $\mathrm{GH}^{(1-3)}$. El factor de crecimiento insulínico (IGF-1) es una proteína producida por diversos órganos, que tiene potentes efectos en el anabolismo y crecimiento celular; es secretada primariamente por el hígado en respuesta a GH. Cada célula en el cuerpo humano puede ser afectada por el IGF-1, pero las células del músculo, cartílago, hueso, hígado, riñón, piel, los pulmones y sistema nervioso periférico tienden a ser más afectados positivamente. Así, GH junto a IGF1, son los más importantes reguladores del crecimiento lineal y del desarrollo del esqueleto, logrando alcanzar la masa ósea pico en la niñez y adolescencia ${ }^{(4-6)}$. El déficit de $\mathrm{GH}$ en niños produce enanismo hipofisario y el exceso de $\mathrm{GH}$ produce gigantismo. En adultos, un exceso produce acromegalia y una deficiencia casi no tiene consecuencias notorias; sin embargo, debe sospecharse en pacientes con cansancio fácil, reducción de la capacidad física, debilidad muscular, incremento de tejido adiposo con distribución centrípeta y alteración del perfil lipídico ${ }^{(5)}$.

Si bien los niveles de GH en suero varían con la edad, siendo muy bajos en la vida fetal, aumentando en la niñez y adolescencia hasta alcanzar niveles constantes en la adultez ${ }^{(7)}$, también existe variabilidad en los niveles de GH de un individuo a otro en una misma etapa de crecimiento; así, por ejemplo, en la etapa prepuberal, las mujeres tienen niveles ligeramente más elevados que los varo- 
nes, y en la adultez ocurre que aquellos de mayor índice de masa corporal, tienen valores más altos de $\mathrm{GH}^{(8-10)}$.

Un factor poco explorado respecto a los niveles sanguineos de hormona de crecimiento es la referente a las altitudes geográficas. Según el último censo realizado en nuestro país, nueve millones de personas viven por encima de los 2000 metros sobre el nivel del mar (msnm); el mayor número de comunidades y ciudades se encuentra entre los 3000 y 5000 msnm. La menor presión atmosférica de la altura condiciona una menor presión parcial de oxígeno y por tanto una menor saturación arterial de oxígeno; en estas condiciones, los procesos fisiológicos ocurren en condiciones de hipoxia crónica. A pesar de estas características, los habitantes nativos de las alturas desarrollan una serie de mecanismos de compensación que son adaptaciones endocrinas que le permiten subsistir desde los primeros días de vida. Con este antecedente, los estudios realizados sobre los niveles plasmáticos de hormona de crecimiento en la altura son discrepantes; mientras algunos investigadores reportan que los nativos de altura tienen mayores concentraciones plasmáticas de $\mathrm{GH}$ que los del nivel del mar ${ }^{(11-15)}$, otros encuentran que los niveles plasmáticos son similares (16-19). Con la finalidad de aportar en este tema, es que nos propusimos realizar el presente trabajo, con el objetivo de determinar los niveles plasmáticos de hormona de crecimiento en sujetos varones, nativos, adultos, a nivel del mar (Lima, $150 \mathrm{msnm}$ ) y a diferentes altitudes en algunas localidades del Perú (Cajamarca: 2750 msnm; Huancayo: $3280 \mathrm{msnm}$; y Morococha: $4540 \mathrm{msnm}$ ).

Al margen de los resultados, es preciso recordar que los niveles altos de GH en el adulto producen acromegalia y los niveles bajos deben ser sospechados en pacientes que han tenido una cirugía o tratamiento radiante por adenoma hipofisario o más frecuentemente en pacientes adultos que presenten cansancio fácil, reducción de la capacidad física, debilidad muscular con reducción de masa muscular, incremento de tejido adiposo con distribución centrípeta y alteración del perfil lipídico, función cardiaca alterada, intolerancia a la glucosa con resistencia a la insulina, osteopenia, entre otros ${ }^{(20,21)}$. También, se ha demostrado que una disminución de la actividad del eje GH/IGF1 puede ser responsable, al menos en parte, de las alteraciones metabólicas que se presentan en pacientes con síndrome de apnea obstructiva del sueño; incrementándose con ello el riesgo de eventos cardiovasculares en estos pacientes ${ }^{(20)}$.

Considerando que la $\mathrm{GH}$ es importante no solo porque interviene en el crecimiento del individuo, sino por sus efectos metabólicos en todas las células del organismo, y que los estudios sobre los niveles de esta hormona en la altura son contradictorios, justificamos la realización del presente estudio con la finalidad de aportar más datos acerca de los eventos fisiológicos que suceden en la altura, siendo el Perú un territorio caracterizado por tener casi una tercera parte de su población viviendo en la altura. Así, nuestros hallazgos contribuirán a una mejor comprensión del sistema endocrino y la fisiología de la hormona de crecimiento en condiciones de hipoxia como son las zonas con mayor altitud geográfica de nuestro país.

\section{MÉTODOS}

Se realizó un estudio observacional, descriptivo, transversal y prospectivo. Se estudió los niveles sanguíneos de hormona de crecimiento de 41 sujetos varones, adultos jóvenes, y sanos de 20 a 35 años de edad, cuya distribución del lugar de residencia fue como sigue: 12 a nivel del mar (Lima 150 msnm), 10 en Cajamarca (2 $750 \mathrm{msnm}$ ), 12 en Huancayo (3 280 msnm) y 7 en Morococha (4 $540 \mathrm{msnm}$ ). Los sujetos que participaron en el estudio fueron residentes habituales de sus loca- lidades, siendo considerados nativos. Se descartó patologías previas y se determinó el buen estado de salud física mediante un examen clínico, incluyendo adecuado índice de masa corporal (IMC). De cada participante se obtuvo los siguientes datos: edad, talla, peso, antecedentes clínicos, IMC. El estudio se realizó en el período 2006 -2008, fue aprobado por el Comité de Ética de la Facultad de Medicina de la Universidad Nacional Mayor de San Marcos y se contó con el debido consentimiento informado de los sujetos de estudio.

Las muestras de sangre fueron tomadas en la vena del antebrazo en condiciones de ayuno y reposo, en las primeras horas de la mañana. La sangre fue centrifugada, el suero separado y congelado a $-20^{\circ} \mathrm{C}$ para su adecuado almacenamiento hasta su posterior análisis en el Instituto de Investigaciones Clínicas de la UNMSM. Se determinó niveles de $\mathrm{GH}$ en suero en $\mathrm{ng} / \mathrm{mL}$ mediante radioinmunoanálisis (RIA), con el uso de kits comerciales, en el que la $\mathrm{GH}$ rotulada con iodo 125 compite con la $\mathrm{GH}$ en la muestra del paciente por sitios sobre el anticuerpo GH específico.

Los resultados son expresados mediante estadística descriptiva. Los datos fueron analizados estadísticamente mediante la prueba t de student, considerando significancia estadística si el valor de $p<0,05$. Se utilizó el programa estadístico SPSS versión 13.

\section{RESULTADOS}

Participaron en el estudio 41 sujetos varones, cuyo promedio de edad fue 28 años (rango: 20 a 35 años). Los valores promedio de $\mathrm{GH}(\mathrm{ng} / \mathrm{mL})$ encontrados

Tabla 1. Hormona de crecimiento de sujetos residentes en diferentes niveles de altitud.

\begin{tabular}{ccccc}
\hline & $\begin{array}{c}150 \mathrm{msnm} \\
(\text { Lima })\end{array}$ & $\begin{array}{c}2750 \mathrm{msnm} \\
\text { (Cajamarca) }\end{array}$ & $\begin{array}{c}3280 \mathrm{msnm} \\
\text { (Huancayo) }\end{array}$ & $\begin{array}{c}4540 \mathrm{msnm} \\
\text { (Morococha) }\end{array}$ \\
\hline $\begin{array}{c}\mathrm{GH} \\
(\mathrm{ng} / \mathrm{mL}) \\
\mathrm{n}\end{array}$ & $0,54 \pm 0,04$ & $0,63 \pm 0,08$ & $1,31 \pm 0,09 *$ & $3,87 \pm 0,16 *$ \\
${ }^{*} p<0,05$. & 12 & 10 & 12 & 7 \\
\hline
\end{tabular}




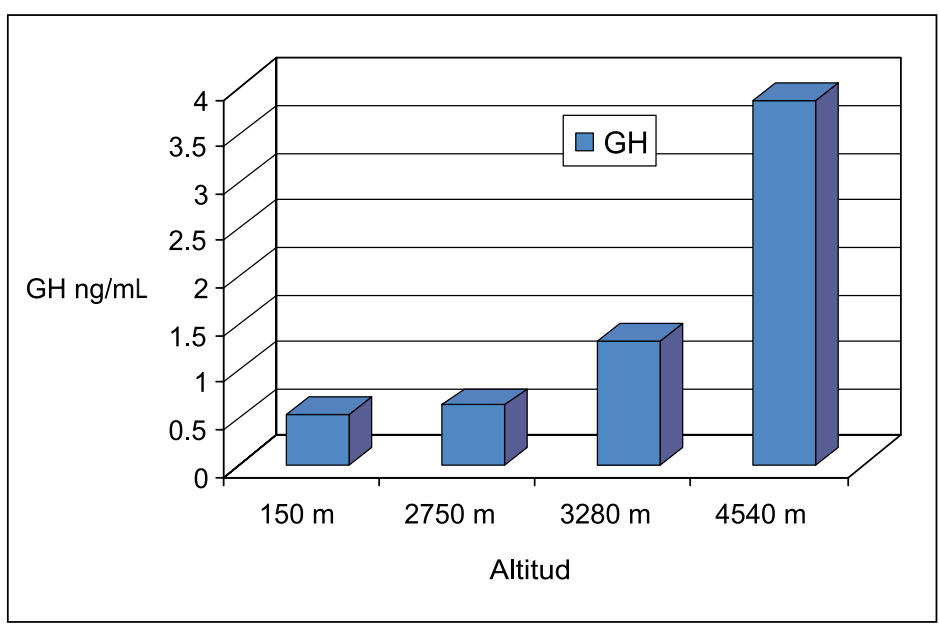

Figura 1. Hormona de crecimiento de sujetos residentes en diferentes niveles de altitud (Lima, 150 msnm; Cajamarca, 2750 msnm; Huancayo, 3280 msnm*; Morococha $\left.4540 \mathrm{msnm}^{*}\right) ;{ }^{*} p<0,05$.

en los sujetos residentes en las diferentes altitudes geográficas estudiadas, son descritos en la tabla 1 y en la figura 1 .

Se halló diferencia significativa entre los valores de $\mathrm{GH}$ encontrados en las alturas de $4540 \mathrm{msnm}$ y $3280 \mathrm{msnm}$, ambas con respecto a los valores de $\mathrm{GH}$ encontrados a nivel del mar (150 msnm) $(p<0,05)$. No se encontró diferencia significativa entre los valores $\mathrm{GH}$ encontrados a $2750 \mathrm{msnm}$ respecto a los valores de $\mathrm{GH}$ encontrados a nivel del mar $(150 \mathrm{msnm})$. No se halló relación entre niveles de $\mathrm{GH}$ y edad de los sujetos de estudio. Para una mejor comprensión de las mediciones halladas, a pesar que los valores normales de $\mathrm{GH}$ varían según los laboratorios, para el presente estudio se consideró valores normales de $\mathrm{GH}$ de $1 \mathrm{ng} / \mathrm{mL}$ a $5 \mathrm{ng} / \mathrm{mL}$.

\section{DISCUSIÓN}

Existen investigaciones acerca de la hormona de crecimiento y su comportamiento en la altura y gran altura (más de 4000 $\mathrm{msnm}$ ), en nativos en condiciones basales o sometidos a actividad física, también en sujetos con exposición aguda o con exposición crónica ${ }^{(22-26)}$. Sin embargo, los resultados son contradictorios. Mientras unos indican aumento en los niveles de $\mathrm{GH}$ en la altura ${ }^{(11-15)}$, otros reportan que los niveles son similares ${ }^{(16-19)}$.
Nuestros resultados muestran niveles de $\mathrm{GH}$ mayores en los sujetos a 3280 msnm (Huancayo) y $4540 \mathrm{msnm}$ (Morococha), respecto a los sujetos a nivel del mar $(p<0,05)$. Algunas investigaciones como las de Franco ${ }^{(17)}$, Villena ${ }^{(19)}$ y Pérez ${ }^{(22)}$, determinaron que los niveles de $\mathrm{GH}$ de nativos de Cerro de Pasco, a 4 $338 \mathrm{msnm}$, fueron similares respecto a los nativos de Lima a $150 \mathrm{msnm}$; resultados parecidos fueron comunicados por Villena en una población del Cusco a 3300 msnm ${ }^{(16)}$. Por el contrario, los trabajos de Richalet ${ }^{(11)}$, Sutton ${ }^{(12,13)}$, Gonzales ${ }^{(14)}$ y más recientemente Benso ${ }^{(15)}$ en sujetos varones aclimatados (no nativos) hallaron niveles mayores de $\mathrm{GH}$ en el suero de sujetos varones a diferentes niveles de altitud con respecto a los varones a nivel del mar; los valores de $\mathrm{GH}$ encontrados en la presente investigación coinciden con el de este grupo de investigaciones. De igual manera, Gonzales y col. realizaron una investigación en varones de Huancayo (3 $280 \mathrm{msnm}$ ) y Cerro de Pasco (4 $340 \mathrm{msnm}$ ), hallando una correlación positiva entre los valores de $\mathrm{GH}$ en suero y el nivel de altitud ${ }^{(14)}$; $\sin$ embargo, no se reportó datos de sujetos nativos que habitaban por debajo de $3380 \mathrm{msnm}$. Nuestro estudio muestra resultados en una población por debajo de los 3000 msnm, como la ciudad de Cajamarca, ubicada a $2750 \mathrm{msnm}$, encontrando que a esta altitud los valores de $\mathrm{GH}$ son similares a los hallados a nivel del mar.

No está claro el mecanismo fisiopatológico del incremento de los niveles de GH en la altura; aún se dispone de poca información al respecto. Sin embargo, Benso y col. ${ }^{(15)}$, quienes determinaron el incremento de los niveles de GH junto a la elevación de los niveles de IGF1 en grandes alturas, proponen que esta hiperactividad del eje $\mathrm{GH} / \mathrm{IGF}-1$ podría deberse a otros fenómenos que también ocurren en la altura, como por ejemplo: anabolismo incrementado de proteínas, adaptaciones de niveles séricos de glucosa (disminución de glucosa en sangre) y metabolismo alterado de lípidos con aumento de lipólisis y ácidos grasos libres en sangre ${ }^{(27,28)}$.

Si bien en nuestra investigación no se determinó niveles de IGF-1, y dado que esta proteína es dependiente de GH, se ha podido decir que IGF-1 también se encuentra elevada en la altura. De hecho, el IGF-1 es el mejor marcador del estado de GH. Así, IGF-1 tendría un efecto importante en el rendimiento físico, anabolismo celular regulador de la división y diferenciación celular, crecimiento óseo y muscular, entre otros, en los sujetos nativos de las alturas en comparación de quienes viven a nivel del mar ${ }^{(15,29)}$.

También se ha determinado que los escaladores aclimatados y bien entrenados en grandes alturas muestran un claro aumento en la concentración media de $\mathrm{GH}$ y esto concuerda con la evidencia que el ejercicio físico constituye un estimulo neuroendocrino que induce la secreción de $\mathrm{GH}^{(15,30)}$. De igual manera, se ha evidenciado una mejor respuesta de $\mathrm{GH}$ a la hormona liberadora de $\mathrm{GH}$ en sujetos que viven a grandes alturas ${ }^{(31)}$. Asimismo, se ha encontrado que los nativos de alturas bajas que se adaptan y aclimatan a grandes alturas, muestran un aumento de $\mathrm{GH}$ más marcado que los sujetos no aclimatados ${ }^{(32)}$.

Si bien ninguno de los sujetos participantes del estudio sobrepasó los $5 \mathrm{ng} / \mathrm{mL}$ de $\mathrm{GH}$, existieron valores por debajo de $1 \mathrm{ng} / \mathrm{mL}$ que, a pesar de un minucioso examen clínico por sospecha de deficiencia de $\mathrm{GH}$, no mostraron debilidad mus- 
cular, ni rendimiento físico disminuido. Consideramos, además, como punto débil de nuestra investigación, el número bajo de sujetos que formaron parte de nuestra población de estudio. Son necesarios estudios futuros sobre este tema en mayor número de sujetos, para una mejor comprensión de este fenómeno.

Concluimos que los niveles de $\mathrm{GH}$ en los sujetos varones, adultos y nativos a $3280 \mathrm{msnm}$ (Huancayo, $\mathrm{n}=12$ ) y $4540 \mathrm{msnm}$ (Morococha, $\mathrm{n}=7$ ) son mayores respecto a los sujetos que viven a 150 (Lima, $n=12$ ).

\section{Agradecimiento}

Al Consejo Superior de Investigaciones de la Universidad Nacional Mayor de San Marcos por la financiación del presente estudio como parte de los proyectos FEDU.

\section{Institución en que se realizó el trabajo:}

Instituto Nacional de Biología Andina. Facultad de Medicina, Universidad Nacional Mayor de San Marcos

\section{Conflictos de intereses:}

Ninguno.

\section{REFERENCIAS BIBLIOGRÁFICAS}

1. Córdova A. Fisiología Dinámica. España: Ed. Elsevier, 2003:552-3.

2. Giustina A, Wehrenberg WB. The role of glucocorticoids in the regulation of growth hormone secretion. Mechanisms and clinical significance. Trends Endocrinol Metab. 1992;3(8):306-11.

3. Tesauro M, Schinzari F, Caramanti M, Lauro R, Cardillo C. Metabolic and cardiovascular effects of ghrelin. Int J Pept. 2010;2010(7). pii: 864342.

4. Ahmed SF, Farquharson C. The effect of GH and IGF1 on linear growth and skeletal development and their modulation by SOCS proteins. J Endocrinol. 2010;206(3):249-59 .

5. Kreitschmann-Andermahr I, Suarez P, Jennings R, Evers N, Brabant G. GH/IGF-I regulation in obesity: mechanisms and practical consequences in children and adults. Horm Res Paediatr. 2010;73:153-60.

6. Svensson J, Tivesten A, Isgaard J. Growth hormone and the cardiovascular function. Minerva Endocrinol. 2005;30(1):1-13.
7. Merimee T, Russel B, Quinn S, Riley W. Hormone and receptor studies: Relationship to linear growth in childhood and puberty. J Clin Endocrinol Metab. 1991;73:1031-7.

8. Gelander L, Ragnar B, Carlsson L, AlbertssonWikland K. Growth hormone-binding protein levels over one year in healthy prepubertal children: Intraindividual variation and correlation with height velocity. Pediatr Res. 1998;43:256-61.

9. Martha JR PM, Rogol AD, Blizzard RM, Shaw MA, Baumann $G$. Growth hormone-binding protein activity is inversely related to 24-hour growth hormone release in normal boys. J Clin Endocrinol Metab. 1991;73:175-81.

10. Martha JR PM, Reiter EO, Davila N, Shaw MA, Holcombe JH, Baumann G. Serum growth hormone-binding protein/receptor: an important determinant of GH responsiveness. J Clin Endocrinol Metab. 1992;75:1464-9.

11. Richalet JP. The endocrine system in high altitude: an exploration of human adaptation. New York, Thomas F. Hormbein, Robert B. Schoene Eds. 2001:627.

12. Sutton JR, Young JD, Lazarow LA y col. The hormonal response to high altitude. Lancet. 1970;2:1194.

13. Sutton J, Young J, Garmendia F. Variaciones hormonales durante el esfuerzo físico en la altura. Arch Biol Andina. 1977;7(2):83-92.

14. Gonzales G, Coyotupa J, Guerra-Garcia R. Elevated levels of growth hormone in natives from high altitude. Interrelationship with glucose levels. Acta Andina. 1992;1 (2):85-8.

15. Benso A, Broglio F, Aimaretti G, Lucatello B, Lanfranco F, Ghigo E, Grottoli S. Endocrine and metabolic responses to extreme altitude and physical exercise in climbers. Eur J Endocrinol. 2007;157(6):733-40.

16. Villena A. Estudio endocrino-metabólico en varones ancianos de Lima (150m) y Cusco (3400m). Acta Andina. 1994;3:29-40.

17. Franco J0, Gonzales GF, Guerra-García R. Niveles basales de hormona de crecimiento en varones residentes de la altura (4340 msnm) y de nivel del mar (150 m). Acta Andina. 1992;1:43.

18. Gonzales $G$. Metabolismo en las grandes alturas. Acta Andina. 2001;9(1-2):31-42.

19. Villena J. Cambios metabólicos en la hipoxia crónica. Acta Andina. 1998;7(2):95-103.

20. Lanfranco F, Motta G, Minetto MA, Ghigo E, Maccario M. Growth hormone/insulin-like growth factor-I axis in obstructive sleep apnea syndrome: an update. J Endocrinol Invest. 2010;33(3):192-6.

21. Hernández A, Jorge R. Deficiencia de la hormona de crecimiento en adultos. Diagnóstico y tratamiento. Rev Cubana Endocrinol. 1999;10(3):212-6.

22. Pérez A. Niveles séricos de hormona de crecimiento e IGF-I y ejercicio físico submáximo en gran altura y a nivel del mar. An Fac Med Lima. 1999;60(1):715.

23.Zhang Y-S, Du J-Z. The response of growth hormone and prolactin of rats to hypoxia. Neurosci Lett. 2000;279:137-40.

24. Gutierrez A, Gonzalez-Gross M, Ruiz JR, Mesa $\mathrm{JL}$, Castillo MJ. Acute exposure to moderate high altitude decreases growth hormone response to physical exercise in untrained subjects. J Sports Med Phys Fitness. 2003;43(4):554-8.

25. Elias AN, Wilson AF, Naqvi S, Pandian MR. Effects of blood $\mathrm{pH}$ and blood lactate on growth hormone, prolactin, and gonadotropin release after acute exercise in male volunteers. Proc Soc Exp Biol Med. 1997;214(2):156-60.

26. Sawhney RC, Malhotra AS. Circadian rhythmicity of growth hormone at high altitude in man. Indian J Physiol Pharmacol. 1991;35(1):55-7.

27. Brooks GA. Increased glucose dependency in circulatory compensated hypoxia. En: Sutton JR, Houston CS \& Coates G. Hypoxia and Mountain Medicine, Eds. Burlington: VA Queen City Printers. 1992:213-6.

28. Roberts AC, Butterfield GE, Cymerman A, Reeves JT, Wolfel EE, Brooks GA. Acclimatization to 4300-m altitude decreases reliance on fat as a substrate. J Applied Physiol. 1996;81:1762-71.

29. Thissen JP, Ketelslegers JM, Underwood LE. Nutritional regulation of the insulin-like growth factors. Endocrine Rev. 1994;15:80-101.

30. Cumming DC. Hormones and athletic performance. En: Felig P, Baxter JD, Frohman LA, Eds. Endocrinology and Metabolism. New York: McGrawHill. 1995:1837-85.

31. Ramirez G, Herrera R, Pineda D, Bittle PA, Rabb $H A$, Bercu BB. The effects of high altitude on hypothalamic-pituitary secretory dynamics in men. Clin Endocrinol. 1995;43:11-8.

32. Heat D, Williams DR. Endocrine function in man at high altitude. London: Churchill Livingston, 1981:247-58.

Manuscrito recibido el 20 de marzo de 2010 y aceptado para publicación el 30 de setiembre de 2010.
Correspondencia:
Elydia Mujica Albán.
Instituto Nacional de Biología Andina
Facultad de Medicina. UNMSM
Hospital Arzobispo Loayza.
Av. Alfonso Ugarte $s / n$.
Lima 1, Perú
Correo electrónico: elymujica@yahoo.es 